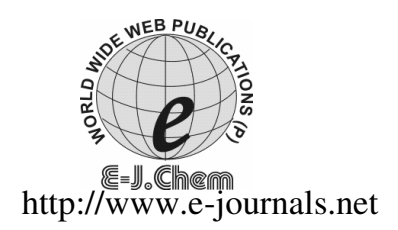

ISSN: 0973-4945; CODEN ECJHAO

E-Journal of Chemistry 2012, 9(1), 145-148

\title{
Synthesis of Certain Poly[bis(benzylidene)] Esters and Investigation on their Photocrosslinkability
}

\author{
D.ROOP SINGH*', S.VASANTHI and J.ARUL MOLI \\ Post-Graduate and Research Department of Chemistry \\ Presidency College (Autonomous), Chennai-600 005, India \\ roopsingh@hotmail.com
}

Received 9 June 2011; Accepted 12 August 2011

\begin{abstract}
Random copolyesters were synthesized directly from respective diacids and diols with diphenylchlorophosphate (DPCP) as a polycondensation agent. These polymers were characterized by FT-IR and NMR spectrometry. Interestingly, they were observed to possess photocrosslinkability which was established using scanning electron microscopy by UV irradiation experiments. Photocrosslinkable polymers have significant applications in the field of nonlinear optics, in the generation of photoresists and photorefractive materials.
\end{abstract}

Keywords: Copolyesters, Polycondensation, Photocrosslinkability, Synthesis

\section{Introduction}

In recent years photocrosslinkable polymers have attracted considerable attention in the research field. These polymers contain photocrosslinkable moieties either in the main chain or as a pendant group in the polymer backbone. The photocrosslinkability of the polymers is due to the presence of photocrosslinkable moieties like azopolyesters ${ }^{1}$, arylidene esters ${ }^{2}$, arylidene ketones, siloxanes ${ }^{3}$, cinnamate esters ${ }^{4,5}$ and acrylate esters ${ }^{6}$. Photocrosslinkable polymers find potential applications in the preparation of photocurable coatings, photocrosslinked hydrogels, optical lithographic materials, photosensitizers, photoresists and photorefractive materials ${ }^{7}$. The controlled crosslinking in photocrosslinkable polymers exhibits their use as information storage devices. Photolinked polymethyl(methacrylate) surface coatings based on poly(ethyleneglycol) have been commercially used as contact lens materials. Polymers having photodimerizable groups find applications in immobilization of enzymes $^{8}$. A series of photocrosslinkable polymers have been synthesized with arylidene keto moiety ${ }^{7,9}$. The present work deals with the synthesis and characterization of certain photocrosslinkable polyesters containing arylidene moiety. The polyesters were synthesized with terephthalic acid, arylidene diol and 2-hydroxyethyl-4-hydroxybenzoate as monomers using diphenylchlorophosphate (DPCP) as the condensing agent. The focus of our work is to investigate the photocrosslinking behaviour of these polyesters. 


\section{Experimental}

Terephthalic acid (T), 4-hydroxybenzaldehyde, vanillin, 4-hydroxybenzoic acid, diphenylchlorophosphate (DPCP) were purchased from Aldrich and used as supplied. LiCl(Merck) was dried at $130{ }^{\circ} \mathrm{C}$ under vacuum for $4 \mathrm{~h}$ and at $180{ }^{\circ} \mathrm{C}$ for $10 \mathrm{~h}$. Cyclohexanone (SRL, India), ethanol and methanol were distilled before use. 2-Chloroethanol (SRL, India) was used as received.

\section{Synthesis of monomers}

\section{Preparation of arylidene diols}

Arylidene diols used in the synthesis of the polyesters were prepared by the condensation of 2 moles of 4-hydroxy-3-methoxybenzaldehyde/4-hydroxybenzaldehyde with one mole of cyclohexanone in the presence of dry methanol and catalytic amount of conc. $\mathrm{H}_{2} \mathrm{SO}_{4}$ as reported by Arumugasamy ${ }^{10}$.

\section{Preparation of 2-hydroxyethyl-4-hydroxybenzoate common diol monomer (D)}

2-Hydroxyethyl-4-hydroxybenzoate monomer is prepared by the condensation of 1 mole of 4-hydroxy benzoic acid and 1 mole of 2-chloroethanol in the presence of potassium bicarbonate in $\mathrm{DMSO}^{11}$.

\section{Copolyester synthesis}

The copolyesters were synthesized by direct polycondensation of two diols and one diacid in the respective mole ratio 1:1:2 using diphenylchlorophosphate (DPCP) as condensing agent in pyridine solution. Thus, the repeat units contain two molecules of the diacid and one molecule of a common diol and one molecule of varying arylidene diol distributed randomly in the polymer chain.

\section{Characterization}

The solubility of the random copolyesters were determined in various solvents qualitatively. About $5 \mathrm{mg}$ of the polyester was added in $1 \mathrm{~mL}$ of the solvent in small stopper test tubes. The mixture was kept for $24 \mathrm{~h}$ with occasional shaking and the polymer solubility was observed. FT-IR spectra of the polyester samples were recorded as $\mathrm{KBr}$ pellet using Perkin Elmer Spectrum One FT-IR Spectrometer from $450 \mathrm{~cm}^{-1}$ to $4500 \mathrm{~cm}^{-1}$. High resolution ${ }^{1}$ HNMR spectra were recorded on a Bruker Biospin FT-NMR spectrometer operating at $400 \mathrm{MHz}$ for ${ }^{1} \mathrm{H}$ and $100 \mathrm{MHz}$ for ${ }^{13} \mathrm{C}$ nucleus in DMSO solvent and with TMS as internal reference. SEM photomicrographs of polyesters were recorded using Hitachi S-3400 SEM instrument.

\section{Results and Discussion}

\section{Synthesis}

The polyesters reported in this work are soluble in high polar solvents like DMSO, DMF, DMAc, acetone and THF. They are insoluble in chlorinated solvents like $\mathrm{CHCl}_{3}$ and $\mathrm{CH}_{2} \mathrm{Cl}_{2}$. The FT-IR spectra (Figure 1) of a typical polyester showed the following characteristic absorption bands that are indicative of aromatic polyesters: FT- $\mathrm{IR}(\mathrm{KBr})$ of poly[ $\{$ bis(4-oxy(benzylidene)cyclohexanone) $\}$-co- $\{1$,4-benzoyl $\}$-co- $\{2$-oxyethyl-4-oxybenzoate $\}]$ (TBHD): 3421(overtone of C=O), 2924, 2853(aliphatic), 1734 (ester carbonyl), 1665 (C=O of cyclohexanone ring), 1599 ( $\mathrm{C}=\mathrm{C}$ aromatic) and 1262 to $1015 \mathrm{~cm}^{-1}$ (C-O-C of ester). ${ }^{1} \mathrm{H}-\mathrm{NMR}$ spectrum of polyester poly[ $\{$ bis(4-oxy(vanillylidene)cyclohexanone) $\}$-co- $\{1,4-$ benzoyl\}-co-\{2-oxyethyl-4-oxybenzoate\}] TVHD recorded in DMSO-d6 is presented in 
Figure 2. The vanillylidene aromatic protons appeared in the region 6.8 to $7.2 \delta$. The methoxy protons of the divanillylidene mesogen appeared as a singlet at $3.9 \delta$. The $\mathrm{OCH}_{2}$ protons connected to the benzoyl group of the common diol D appeared at $4.7 \delta$. The multiplets in the range of 7.4 to $8.4 \delta$ referred to benzoyl aromatic protons. The protons of cyclohexanone appeared at 1.8 at $2.9 \delta$.

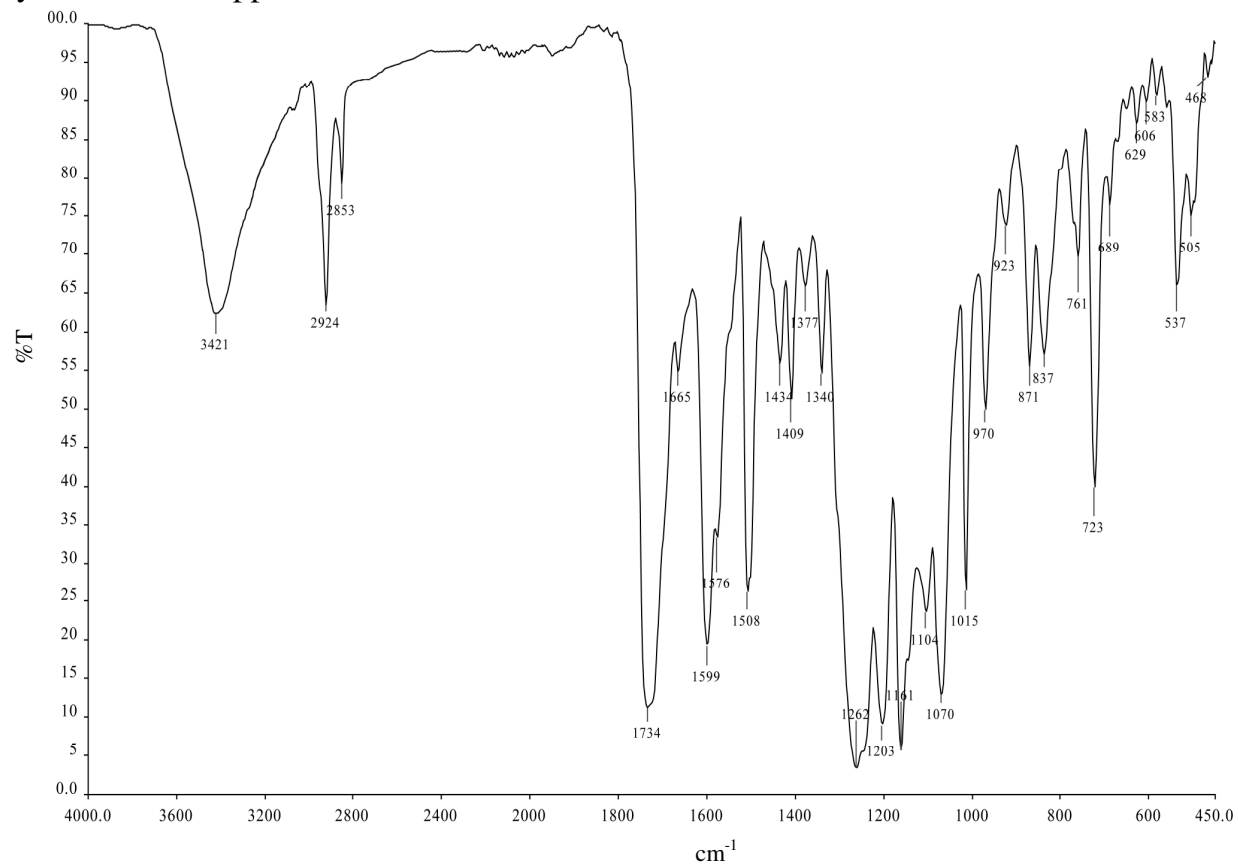

Figure 1. FT-IR spectrum of polyester TBHD

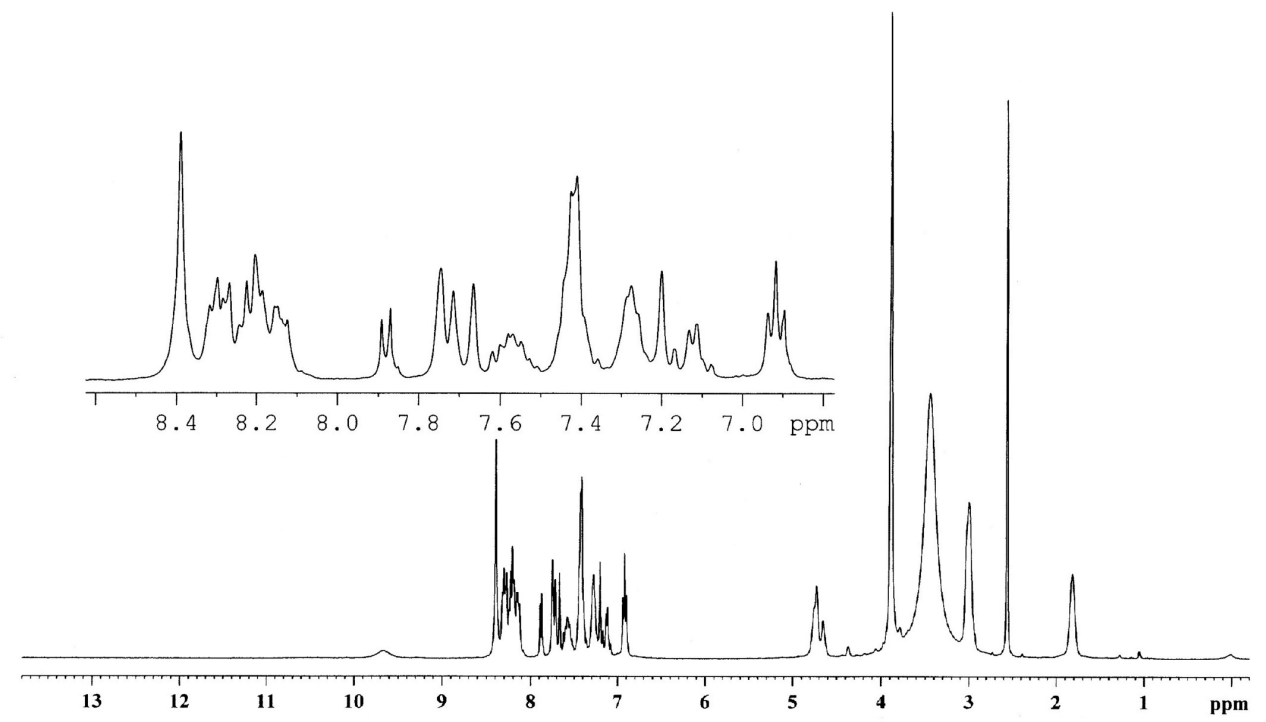

Figure 2. ${ }^{1} \mathrm{H}$ NMR spectrum of polyester TVHD 


\section{Photocrosslinkability studies}

The polyesters prepared contain arylidene moiety, which functions as photo-active chromophore. A small quantity of about $2 \mathrm{mg}$ of the finely powdered polyester samples were exposed to UV irradiation under $160 \mathrm{~W}$ medium pressure mercury lamp in air for $5 \mathrm{~h}$ and the SEM photomicrographs before and after UV irradiation for a typical copolyester (TBHD) are shown in Figure 3. It is evident from these photographs that there is photocrosslinking in the polyester after UV irradiation. The photocrosslinking is attributed through 2+2 cycloaddition of carbon-carbon double bond in the bis(benzylidene)/ bisvanillylidenecycloalkanone units present in the polymer backbone.
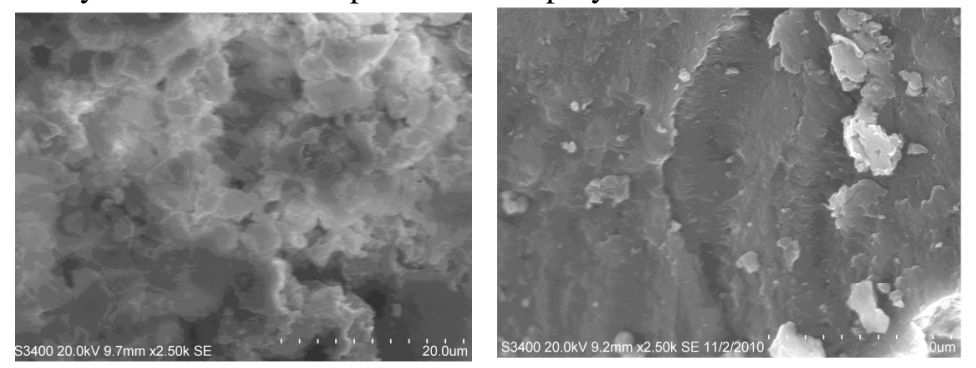

Figure 3. (a) TBHD before UV irradiation (b) TBHD after UV irradiation

\section{Conclusion}

Random photocrosslinkable polyesters containing arylidene keto moiety in the main chain were synthesized and characterized spectroscopically. These polyesters can be crosslinked by UV irradiation, which was established by SEM investigation. The potential group responsible for such photocrosslinking is the arylidene keto moiety which could be useful in developing optical information storage devices.

\section{References}

1. Acinerno D, Amendola E, Fresa R, Ianne I P and Vacca P, Polym., 2000, 41, 7785.

2. Kannan P and Murugavel S C, J Polymn Sci Part A: Polym Chem., 1999, 37, 3285.

3. Racles C, Cozan V, Cazacu M, High Performance Polym., 2003,15, 231.

4. Hyder Ali A and Srinivasan K S V, J Appl Polym Sci., 1998, 67, 441-448.

5. Kawatsuki N, Takatsuka H, Yamamoto T and Sangen O, J Polym Sci Part A Polym Chem., 1998, 36, 1521-1526.

6. Hageman H J, Eur Polym J., 1999, 35, 991.

7. Gangadhara and Kishore K, Macromolecules, 1993, 26, 2995-2996.

8. Koch T, Ritter H and Buchholz N, Makromol Chem., 1989, 190, 1369.

9. Khairou K S, Abdullah M A, Aly K I, Nahas N M and Al-Bonoan A M, Arabian J Chem., 2009, 2 1103-1112.

10. Arumugasamy E, Ph.D., Thesis, University of Madras, 1998.

11. Angeloni A S, Laus M, Chiellini E, Galli G and Francescangeli O, Eur Polym J., 1995, 31, 254. 


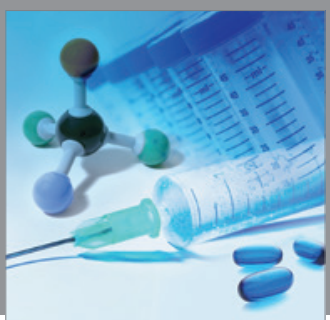

International Journal of

Medicinal Chemistry

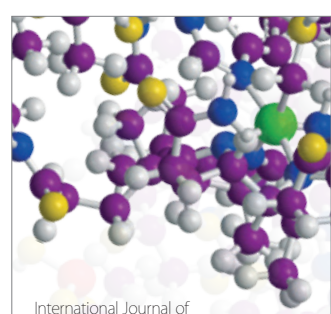

Carbohydrate Chemistry

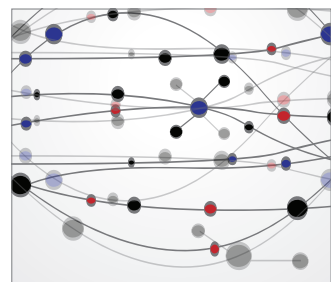

The Scientific World Journal
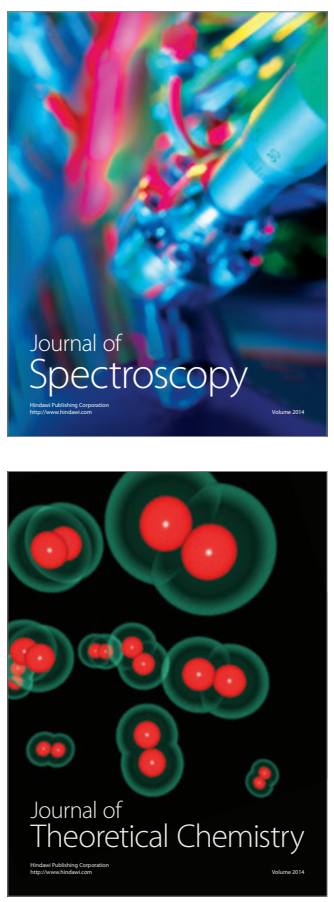
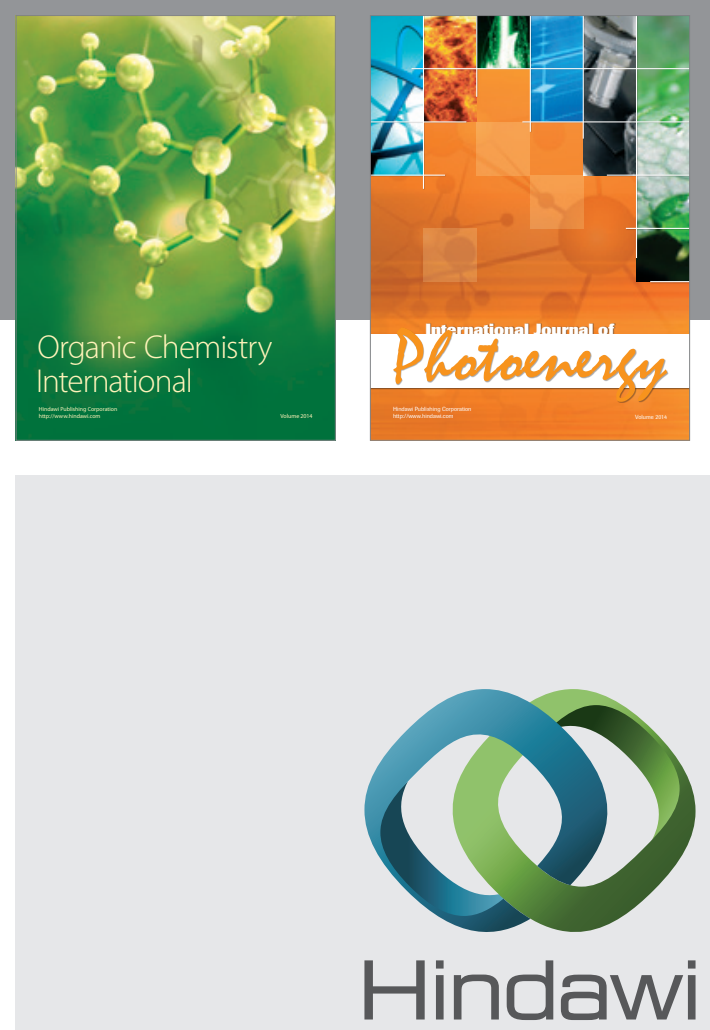

Submit your manuscripts at

http://www.hindawi.com
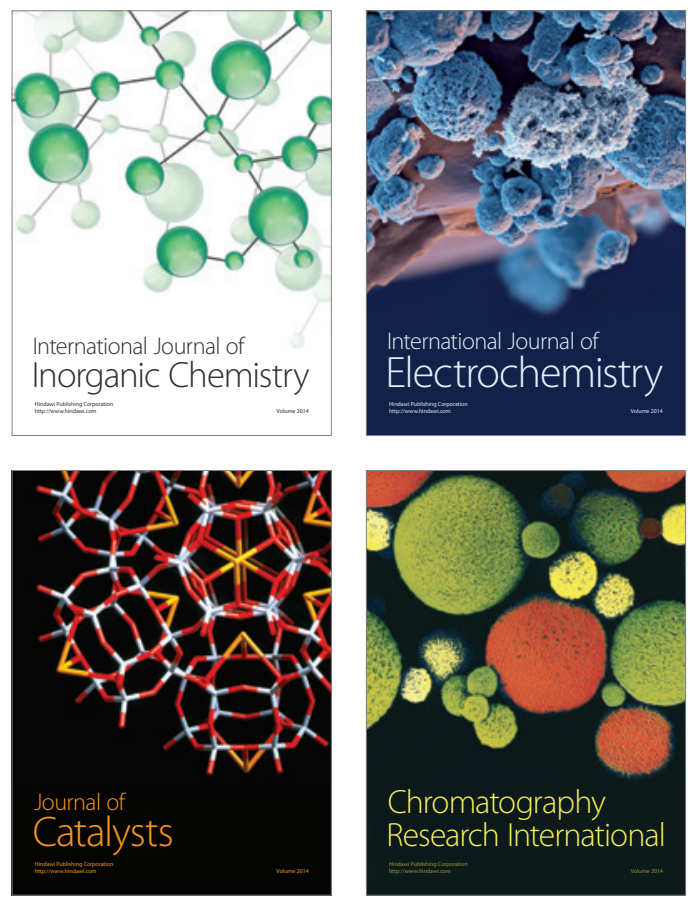
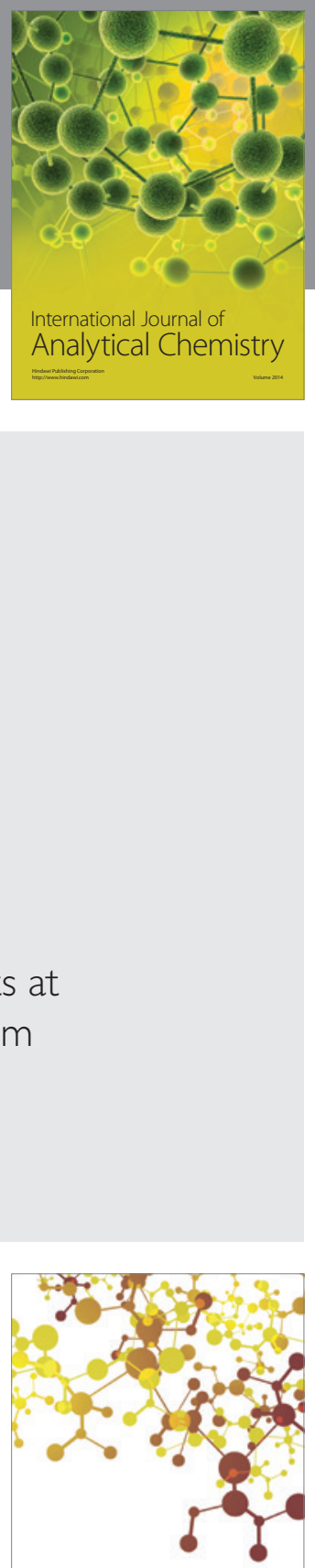

Journal of

Applied Chemistry
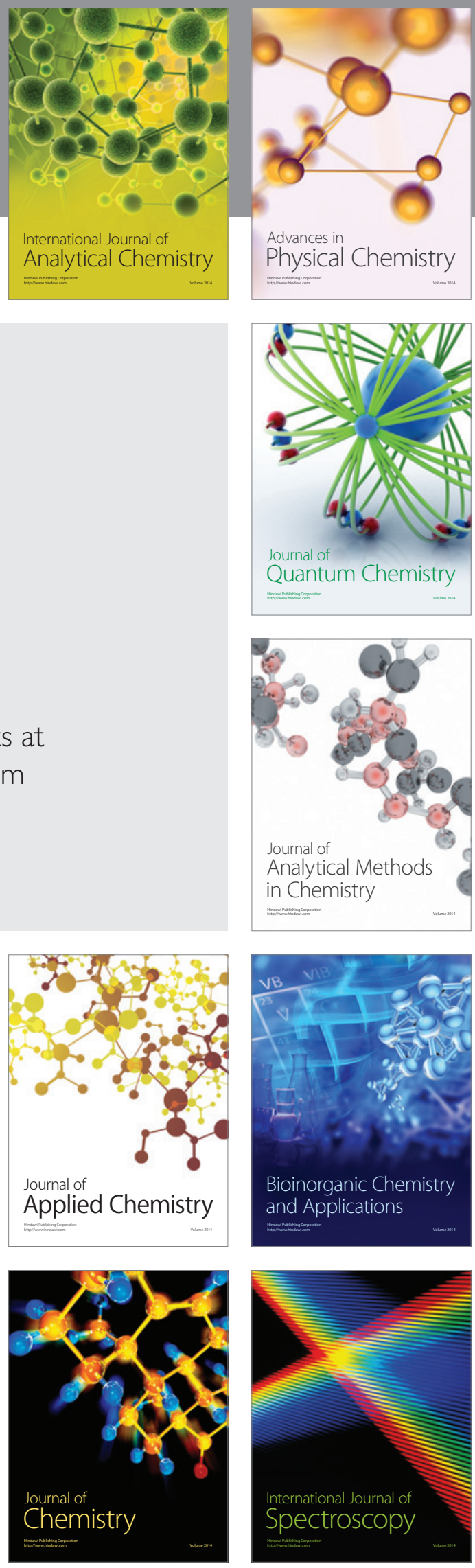